\title{
Iron supplementation to treat anaemia in adult critical care patients: a systematic review and meta-analysis
}

\author{
Akshay Shah ${ }^{1 *}$ (D), Noémi B. Roy ${ }^{2}$, Stuart McKechnie ${ }^{1}$, Carolyn Doree ${ }^{3}$, Sheila A. Fisher ${ }^{3}$ and Simon J. Stanworth ${ }^{4,5}$
}

\begin{abstract}
Background: Anaemia affects $60-80 \%$ of patients admitted to intensive care units (ICUs). Allogeneic red blood cell (RBC) transfusions remain the mainstay of treatment for anaemia but are associated with risks and are costly. Our objective was to assess the efficacy and safety of iron supplementation by any route, in anaemic patients in adult ICUs.

Methods: Electronic databases (CENTRAL, MEDLINE, EMBASE) were searched through March 2016 for randomized controlled trials (RCT)s comparing iron by any route with placebo/no iron. Primary outcomes were red blood cell transfusions and mean haemoglobin concentration. Secondary outcomes included mortality, infection, ICU and hospital length of stay, mean difference (MD) in iron biomarkers, health-related quality of life and adverse events.

Results: Five RCTs recruiting 665 patients met the inclusion criteria; intravenous iron was tested in four of the RCTs. There was no difference in allogeneic RBC transfusion requirements (relative risk 0.87, $95 \%$ confidence interval (CI) 0.70 to $1.07, p=0.18$, five trials) or mean number of RBC units transfused (MD $-0.45,95 \% \mathrm{Cl}-1.34$ to $0.43, p=0.32$, two trials) in patients receiving or not receiving iron. Similarly, there was no difference between groups in haemoglobin at short-term (up to 10 days) (MD $-0.25,95 \% \mathrm{Cl}-0.79$ to $0.28, p=0.35$, three trials) or mid-term follow up (last measured time point in hospital or end of trial) (MD $0.21,95 \% \mathrm{Cl}-0.13$ to $0.55, p=0.23$, three trials). There was no difference in secondary outcomes of mortality, in-hospital infection, or length of stay. Risk of bias was generally low although three trials had high risk of attrition bias; only one trial had low risk of bias across all domains.

Conclusion: Iron supplementation does not reduce RBC transfusion requirements in critically ill adults, but there is considerable heterogeneity between trials in study design, nature of interventions, and outcomes. Well-designed trials are needed to investigate the optimal iron dosing regimens and strategies to identify which patients are most likely to benefit from iron, together with patient-focused outcomes.
\end{abstract}

Trial registration: PROSPERO International prospective register of systematic reviews CRD42015016627. Registered 2 March 2015.

Keywords: Anaemia, Transfusion, Red blood cell, Iron, Haemoglobin, Meta-analysis

\section{Background}

Anaemia is common in critically ill patients and is associated with adverse outcomes $[1,2]$. Observational studies have shown that anaemia affects $60-80 \%$ of patients admitted to critical care and is present at critical care discharge in $75 \%$ of patients [3, 4]. The implementation

\section{* Correspondence: akshayshah@doctors.org.uk}

${ }^{1}$ Nuffield Department of Anaesthetics, Level 2 John Radcliffe Hospital, Oxford

University Hospitals NHS Foundation Trust, Oxford, UK

Full list of author information is available at the end of the article of restrictive red cell transfusion policies is likely to compound the observed high prevalence of anaemia [5]. Allogeneic red blood cell (RBC) transfusion has been the mainstay of treatment for critical illness anaemia, although studies suggest that patients who receive allogeneic RBC transfusion are at increased risk of mortality, ischaemic complications, delayed wound healing, multiorgan dysfunction and increased length of stay [6-9].

Interest has now focused on the identification of irondeficiency as an aetiological factor contributing to the anaemia observed in critical illness. However, diagnosis 
of absolute iron-deficiency continues to be challenging in critical care. Critically ill anaemic patients typically display the hallmarks of anaemia of inflammation and iron-restricted erythropoiesis secondary to functional iron deficiency, absolute iron deficiency or iron sequestration [10]. As for other inflammatory conditions, hepcidin as a key regulator of iron homeostasis is considered to contribute to this functional iron deficiency in critically ill patients [10]. Iron supplementation, whether oral or intravenous (iv), is an effective treatment for patients with absolute iron deficiency; however, iron is essential for bacterial growth, and exogenous intravenous iron administration has been reported to be associated with increased risk of infection, raising significant safety concerns, particularly in this patient group [11].

To assess current knowledge of the role of iron supplementation in critical care, we performed a systematic review to evaluate the effect of iron supplementation on allogeneic RBC transfusion requirements and clinical outcomes (mortality, quality of life and infection) in this patient group. The safety of iron supplementation was also evaluated.

\section{Methods}

Study methods were implemented according to a predefined protocol [12].

\section{Study identification}

Trials were identified from database searches to 14 March 2016 of CENTRAL (The Cochrane Library Issue 2, 2016), MEDLINE (1946 onwards), Embase (1974 onwards), CINAHL (1937 onwards), PubMed (E-publications ahead of print only), Web of Science (1990 onwards) and the Transfusion Evidence Library (1950 onwards). We searched for ongoing trials using the trial registration websites World Health Organization International Clinical Trials Registry Platform and ClinicalTrials.gov. We did not restrict our search for trials by date, language, or publication status. The full search strategy is shown in Additional file 1.

\section{Eligibility criteria}

To be included in this systematic review, the following criteria had to be met: (1) study design: randomized controlled trials; (2) patients: all patients admitted to any adult intensive care units (ICUs) or high-dependency units (HDUs); and (3) intervention: iron supplementation by any route (enteral, iv, intramuscular) versus placebo or no iron therapy; co-interventions were only included if they were present in both/all treatment arms. Trials including children, pregnant women and patients with chronic kidney disease were excluded $[13,14]$.

\section{Study selection and data extraction}

Two reviewers (AS, NR) independently screened citations that met inclusion criteria and extracted data onto a pre-piloted data collection form. Disagreements were solved through discussion and if no resolution was found a third author (SS) adjudicated. Two reviewers (AS and SS) independently extracted study characteristics and outcomes, including study design, methodology, patient characteristics, iron supplementation strategies and outcome data. We sought unclear or missing data by contacting the authors of the individual trials and allowing them 28 days to respond.

\section{Study outcomes}

The primary outcomes of this systematic review were: (1) requirement for allogeneic RBC transfusion; (2) mean number of RBCs transfused; and (3) mean haemoglobin concentration. Secondary outcomes were: (1) all-cause mortality; (2) in-hospital infection as defined in individual trials (e.g. confirmed/suspected diagnosis and antibioticfree days); (3) mean ICU length of stay (LOS); (4) mean hospital LOS; (5) mean measurement of biomarkers of iron status, e.g. ferritin, erythrocyte zinc protoporphyrin (eZPP), transferrin saturation (Tsat) and serum iron; (6) morbidity scales (sequential organ failure assessment (SOFA) score and multiple organ dysfunction score (MODS)); and (7) health-related quality of life (HrQoL) (e.g. SF-36) and serious adverse events defined as "any untoward medical occurrence that at any dose results in death, is life-threatening, requires in-patient hospitalisation or prolongation of existing hospitalization, or results in persistent or significant disability/incapacity" [15]. We defined two time points: (1) short-term (up to 10 days) and (2) mid-term (the last measured time point in hospital or end of the trial).

\section{Risk of bias assessment}

Study quality was judged using the Cochrane Collaboration tool for assessing risk of bias [16]. We assessed the following domains for each study: random sequence generation, allocation concealment, blinding of participants, personnel and outcome assessors, incomplete outcome data, selective reporting and other bias such as financial conflicts of interest. A three-point scale was used to determine low, high or unclear risk of bias.

\section{Data synthesis}

Statistical analyses were performed using Review Manager [17]. Random effects models were used throughout due to the likely heterogeneity arising from different iron supplementation strategies used and different ICU settings. Where possible, continuous variables were reported as mean difference (MD) with $95 \%$ confidence interval (CI) at follow-up time points as defined above. For outcomes 
which were deemed likely to be skewed in distribution, namely number of RBC transfusions and serum ferritin, reported mean and standard deviation were transformed to a natural logarithmic scale using the methods of Higgins et al. [18]. In one study that reported count data for the number of transfusions [19], raw data values were log transformed after adding a constant of 0.5 to each value due to the number of patients who received no transfusions. Dichotomous variables were reported as relative risk (RR) with corresponding $95 \%$ CI. Heterogeneity was assessed using the $I^{2}$ statistic [20]. In one trial with two intervention arms, data were pooled across the two intervention arms and compared with the single control group for the main analysis [19].

\section{Subgroup and sensitivity analysis}

Subgroup analyses were performed if there were at least two trials with comparable subgroups reporting the primary outcomes: (1) different iron preparations (enteral vs intravenous) and (2) use of co-interventions, e.g. erythropoietin. Sensitivity analyses for risk of bias were intended but were not carried out due to the limited number of included studies.

\section{Results}

Our search identified 894 references. Of these, 32 underwent full-text screening after exclusion by screening of titles, duplicates and abstracts (see Preferred Reporting Items for Systematic Reviews and MetaAnalyses (PRISMA) study flow diagram in Fig. 1). Five studies were included in the final analysis [19, 21-24]. One ongoing trial was identified [25].

\section{Description of included studies}

Patient populations, trial interventions and outcomes are summarised in Table 1 . The five studies included a total of 665 randomised participants of whom 368 received iron and 297 received no iron/placebo. Four trials were carried out in surgical ICUs (cardiothoracic, trauma, general surgical, burns and neurosurgical) and one trial was carried out in a mixed ICU that included medical, surgical and trauma patients. Two trials compared intravenous iron to intravenous placebo, one trial compared intravenous iron to no iron (control) and one trial compared oral iron to oral placebo. A fifth trial included three arms: (1) intravenous iron and oral placebo, (2) oral iron and intravenous placebo, and (3) oral and intravenous placebo. Co-interventions were given in two trials: ascorbic acid, cyanocobalamin and folic acid [22] or folic acid only [24]. All trials reported different dosing regimens as described in Table 1.

\section{Quality assessment and Risk of Bias}

The risk of bias in individual trials is shown in Fig. 2. Only one trial had low risk of bias in every domain [19].
Three trials reported high levels of attrition of randomized participants. In one trial, only participants who received all six possible doses of the study drug were analysed for haemoglobin and other markers of iron status (46/75 participants in the intervention groups and $48 / 75$ participants in the control group) [23], in the second trial, 17 participants in the intervention group and 7 participants in the control group were excluded from the final analysis [19] and in the third trial only patients who had not received a blood transfusion were analysed [21].

\section{Effect of interventions}

\section{Allogeneic red blood cell transfusion}

All five trials reported on the number of patients who received an allogeneic $\mathrm{RBC}$ transfusion. In meta-analysis there was no evidence that iron supplementation to treat anaemia in critical care patients reduced allogeneic RBC transfusion requirements (RR $0.88,95 \% \mathrm{CI} 0.74$ to 1.06 ; $p=0.18, I^{2}=46 \%$ ) (Fig. 3a). In subgroup analysis by route of administration there was no evidence of an effect of iron supplementation in patients who received oral iron or those who received intravenous iron (Fig. 4).

\section{Mean number of RBC units}

Only two trials reported the number of RBC units received in both groups $[19,24]$. After transforming to a $\log$ scale, meta-analysis of these two trials showed no evidence that iron supplementation reduced the mean number of allogeneic RBC units transfused (MD -0.45, $95 \%$ CI -1.34 to $0.43, p=0.32, I^{2}=79 \%$ ) (Fig. 3b).

\section{Mean difference in haemoglobin concentration}

Mean (and standard deviation (SD)) changes in haemoglobin concentration at short-term and mid-term follow-up time points was reported in three trials [19, 21, 24]. There was no significant difference in the mean haemoglobin concentration between patients who received iron and those who did not, either at short-term (MD - $0.25,95 \%$ CI -0.79 to $0.28, p=0.35, I^{2}=57 \%$ ) (Fig. 3c) or mid-term follow-up (MD 0.21, $95 \%$ CI -0.13 to $0.55, p=0.23, I^{2}=$ $0 \%$ ) (Fig. 3d). In the two trials that did not report mean and SD, one trial reported no change in haematocrit concentration over a period of 28 days in both groups [22] and the other trial found no significant differences in haemoglobin concentration between groups at any followup time point over 14 days [23].

\section{In-hospital mortality}

Four trials reported in-hospital mortality as an outcome [21-24]. In meta-analysis there was no difference in the risk of mortality between patients who received iron supplementation and those who did not (RR 1.04, $95 \%$ 


\section{PRISME PRISMA 2009 Flow Diagram}

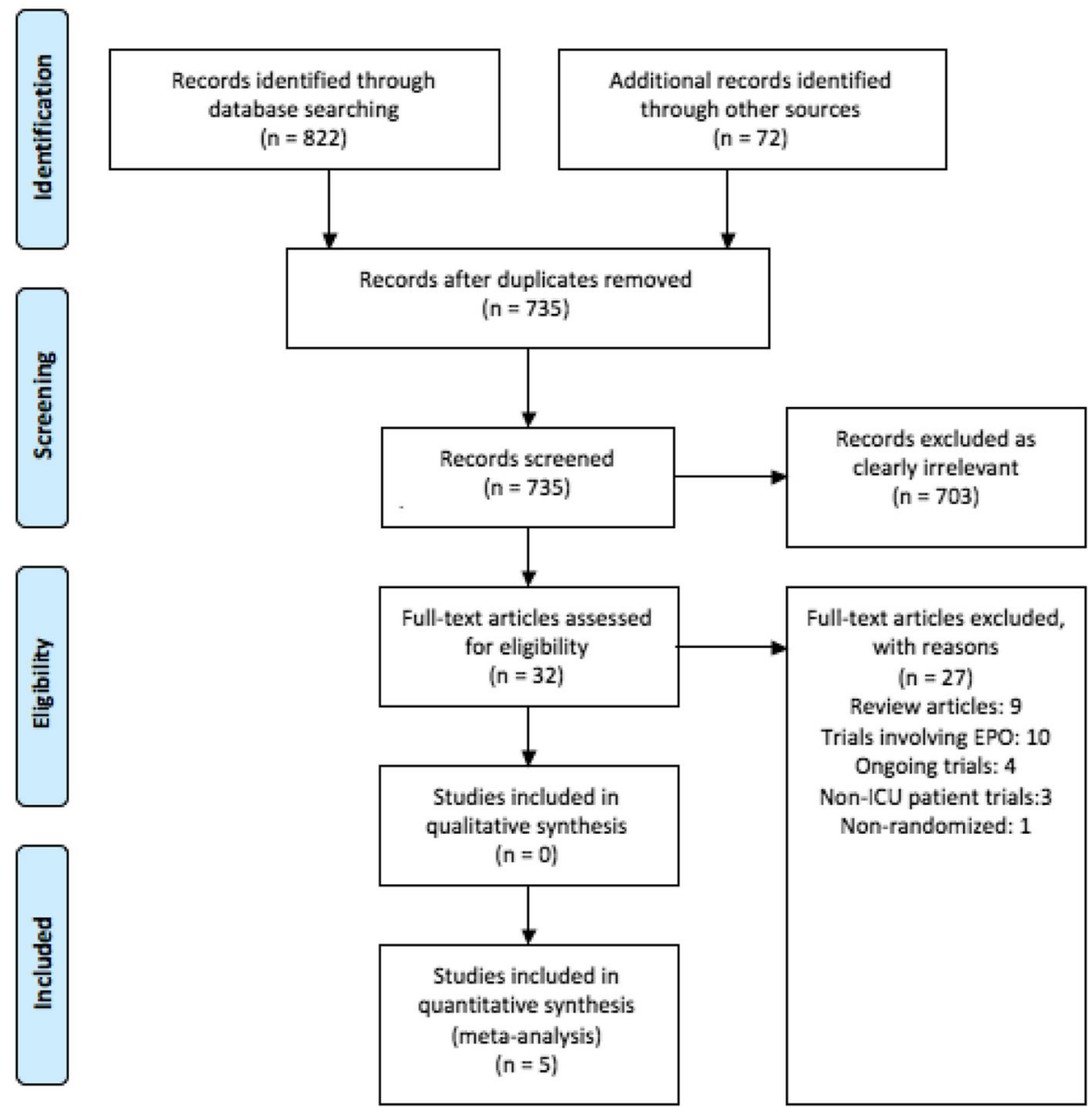

Fig. 1 Preferred Reporting Items for Systematic Reviews and Meta-analyses (PRISMA) flow chart showing the selection of studies in this meta-analysis. EPO erythropoietin

CI 0.43 to $2.52 ; p=0.92, I^{2}=52 \%$ ) (Fig. 5a). Causes of mortality were not reported in any trials.

\section{In-hospital infection}

Two studies provided data on infection and antibiotic usage $[22,23]$. There was no evidence of a difference in the risk of infection in patients who received iron supplementation and those who did not (RR 0.89, $95 \% \mathrm{CI}$ 0.74 to $1.08, p=0.23, I^{2}=0 \%$ ) (Fig. $5 \mathrm{~b}$ ). Neither study found a significant difference in mean number of antibiotic days between the intervention and control groups ( 14 vs. 16 days, $p=0.45 ; 14$ vs. 16 days, $p=0.64$ ).

\section{Length of stay}

Three trials reported ICU LOS [22-24] and two trials additionally reported hospital LOS $[22,23]$. One trial reported a significantly longer ICU LOS in the control group compared to the intervention group (58+/- 31 vs. $29+/-18$ days, $p<0.05$ ) [24], whereas two trials found no difference in ICU LOS (12 vs. 14 days, $p=0.69 ; 10$ vs. 11 days, $p=0.53)[22,23]$. Similarly, these trials found no differences in hospital LOS (14 vs. 16 days, $p=0.24$; 14 vs. 16 days, $p=0.50)$ [22, 23]. Meta-analysis was not performed as only one trial provided mean and SD values.

\section{Biomarkers of iron status}

All trials reported serum ferritin measurements. Three trials reported mean and SD data [19, 21, 24] which were log-transformed due to the likely skewing of this outcome. Iron supplementation was associated with higher log serum ferritin levels at both short-term (MD $0.41 \mathrm{ng} / \mathrm{mL}, 95 \%$ CI 0.06 to $\left.0.77, p=0.02, I^{2}=78 \%\right)$ (Fig. 5c) and mid-term follow up (MD $0.68 \mathrm{ng} / \mathrm{mL}, 95 \%$ CI 0.53 to $0.83, p<0.00001, I^{2}=0 \%$ ) (Fig. $5 \mathrm{~d}$ ). In two 
Table 1 Characteristics of included studies $(n=5)$

\begin{tabular}{|c|c|c|c|c|c|c|c|c|c|c|}
\hline & \multicolumn{10}{|l|}{ Study } \\
\hline & \multicolumn{2}{|c|}{ Garrido-Martin et al. (2012) [19] } & \multicolumn{2}{|c|}{$\begin{array}{l}\text { Madi-Jebara et al. (2004) } \\
\text { [21] }\end{array}$} & \multicolumn{2}{|c|}{ Pieracci et al. (2009) [22] } & \multicolumn{2}{|c|}{ Pieracci et al. (2014) [23] } & \multicolumn{2}{|c|}{ van Iperen et al. (2000) [24] } \\
\hline & Iron & No iron & Iron & No iron & Iron & No iron & Iron & No iron & Iron & No iron \\
\hline Randomised, $n$ & iv 71 , oral 73 & 66 & 40 & 40 & 97 & 103 & 75 & 76 & 12 & 12 \\
\hline Analysed $n$ & iv 54 , oral 53 & 52 & 40 & 40 & 97 & 103 & 75 & 75 & 12 & 12 \\
\hline Age, mean (SD/range) & $\begin{array}{l}\text { iv } 65(11) \\
\text { oral } 65(10)\end{array}$ & $65(12)$ & $59.1(9.1)$ & $55.3(9.5)$ & $55.7(1.9)$ & $58.2(1.7)$ & $41.6(18-83)$ & $40.4(18-87)$ & $67(49-89)$ & $69(45-80)$ \\
\hline Male, \%: & $\begin{array}{l}\text { iv } 70.3 \% \\
\text { oral } 71.7 \%\end{array}$ & $76.9 \%$ & $90 \%$ & $90 \%$ & $50.5 \%$ & $46.6 \%$ & $77.3 \%$ & $60.5 \%$ & $66.6 \%$ & $66.6 \%$ \\
\hline ICU setting & \multicolumn{2}{|l|}{ Cardiothoracic } & \multicolumn{2}{|c|}{ Cardiothoracic } & \multicolumn{2}{|c|}{$\begin{array}{l}\text { General surgical, burns, } \\
\text { neurosurgical }\end{array}$} & \multicolumn{2}{|l|}{ Trauma } & \multicolumn{2}{|c|}{$\begin{array}{l}\text { Mixed (surgical, medical, } \\
\text { neurological, trauma) }\end{array}$} \\
\hline Inclusion criteria & \multicolumn{2}{|c|}{$\begin{array}{l}\text { Age }>18 \text { years old, elective } \\
\text { cardiac surgery under } \\
\text { extracorporeal circulation, no } \\
\text { previous anaemia, susceptible } \\
\text { to treatment, no preoperative } \\
\text { blood transfusion, able to } \\
\text { complete all study visits as per } \\
\text { protocol, able to provide } \\
\text { written consent }\end{array}$} & \multicolumn{2}{|c|}{$\begin{array}{l}\text { Elective cardiac surgery } \\
\text { with CPB, post-pump } \mathrm{Hb} \\
7-10 \text { g.dL }\end{array}$} & \multicolumn{2}{|c|}{$\begin{array}{l}\text { General surgical, burn, } \\
\text { neurosurgical ICUs, age } \\
>18 \text { years, } \mathrm{Hb}<13 \mathrm{~g} . \mathrm{dL}^{-1} \\
\text { prior to enrollment, }< \\
72 \text { hours from hospital } \\
\text { admission, current tolerance } \\
\text { of enteral medication, } \\
\text { expected ICU LOS }>5 \text { days }\end{array}$} & \multicolumn{2}{|c|}{$\begin{array}{l}\text { Admitted to ICU with trauma, } \\
\mathrm{Hb}<12 \mathrm{~g}^{-\mathrm{dL}^{-1}} \text {, Age }>18 \text { years, }< \\
72 \text { hours from ICU admission, } \\
\text { expected ICU LOS }>5 \text { days }\end{array}$} & \multicolumn{2}{|c|}{$\begin{array}{l}\mathrm{Hb}<11.2 \mathrm{~g}^{\mathrm{dL} L^{-1}},<12.1 \mathrm{~g} \cdot \mathrm{dL}^{-1} \text { if } \\
\text { cardiac disease, age }>18 \text { years, } \\
\text { expected ICU LOS }>7 \text { days, } \\
\text { informed consent from patient } \\
\text { or relative }\end{array}$} \\
\hline Exclusion criteria & \multicolumn{2}{|c|}{$\begin{array}{l}\text { Elective cardiac surgery without } \\
\text { exclusion criteria, fibrinolytic } \\
\text { therapy } 48 \text { hours prior to CPB, } \\
\text { impaired renal function } \\
\left(C^{-1} C l<50 \text { mls.min }^{-1}\right) \text {, previous } \\
\text { surgery for IE, repeat surgery, } \\
\text { pregnant or lactating, active } \\
\text { gastrointestinal bleeding, B12 } \\
\text { deficit, ferropenic anaemia, } \\
\text { asthma or allergy, active } \\
\text { infection, included in another } \\
\text { study, hepatic disease, history of } \\
\text { allergy to iron, unlikely to } \\
\text { adhere to protocol follow up }\end{array}$} & \multicolumn{2}{|c|}{$\begin{array}{l}\text { Intra-operative blood } \\
\text { transfusion, post-operative } \\
\text { haemodynamic instability, } \\
\text { ejection fraction < } 40 \% \text {, } \\
\text { chronic kidney disease, } \\
\text { hypothermic bypass, } \\
\text { hypersensitivity to iron }\end{array}$} & \multicolumn{2}{|c|}{$\begin{array}{l}\text { Active bleeding, chronic } \\
\text { inflammatory conditions, } \\
\text { end-stage renal disease, } \\
\text { haematologic disorders, } \\
\text { macrocytic anaemia, current } \\
\text { use of EPO, pregnancy, } \\
\text { prohibition of RBC transfusion, } \\
\text { imminent death, co- } \\
\text { enrollment in another trial }\end{array}$} & \multicolumn{2}{|c|}{$\begin{array}{l}\text { Active haemorrhage, iron } \\
\text { overload (serum ferritin > } \\
1000 \mathrm{ng}^{-1} \mathrm{~mL}^{-1} \text { ), conditions } \\
\text { associated with iron overload } \\
\text { e.g. haemachromatosis, active } \\
\text { infection, chronic inflammatory } \\
\text { conditions, pre-existing } \\
\text { haematological disorders, } \\
\text { macrocytic anaemia, current/ } \\
\text { recent (within } 30 \text { days) use of } \\
\text { immunosuppression, use of EPO } \\
\text { within } 30 \text { days, pregnancy or } \\
\text { lactation, prohibition of RBCs, } \\
\text { imminent death, history of } \\
\text { allergy to iron }\end{array}$} & \multicolumn{2}{|c|}{$\begin{array}{l}\text { Pregnancy, iron deficiency } \\
\text { anaemia (ferritin }<50 \text { ug..-1), } \\
\text { vitamin B12 deficiency }(<160 \\
\text { pmol..-1 }) \text {, recent use of } \\
\text { cytostatics or recent } \\
\text { radiotherapy, life expectancy < } \\
7 \text { days, chronic renal failure, } \\
\text { prior use of EPO }\end{array}$} \\
\hline Intervention(s) & \multicolumn{2}{|c|}{$\begin{array}{l}\text { (1) Iron-hydroxide sucrose } \\
\text { complex, iv (Venofer; Uriach } \\
\text { Laboratory) } 3 \text { doses of } 100 \\
\text { mg/24 hours during pre- } \\
\text { and post-hospitalisation, and } \\
1 \text { pill/ } 24 \text { hours of oral placebo } \\
\text { during the same period and } \\
\text { during } 1 \text { month after discharge } \\
\text { (2) Ferrous fumarate ( } 105 \text { mg of } \\
\text { iron) } 1 \text { pill/ } 24 \text { hours orally pre- }\end{array}$} & \multicolumn{2}{|c|}{$\begin{array}{l}\text { i) Iron, iv (Venofer; } \\
\text { Luitpold Pharmaceuticals) } \\
200 \text { mg/day to reach total } \\
\text { iron deficit + s/c placebo (a) }\end{array}$} & \multicolumn{2}{|c|}{$\begin{array}{l}\text { Enteral ferrous sulphate } \\
325 \mathrm{mg} \text { (oral solution or } \\
\text { capsule) (Rockwell } \\
\text { Compounding Inc.) thrice } \\
\text { daily until hospital } \\
\text { discharge. Co-intervention: } \\
\text { ascorbic acid } 500 \text { mg thrice } \\
\text { daily, cyanocobalamin } 1 \mathrm{mg} \text {, } \\
\text { folic acid } 1 \mathrm{mg}\end{array}$} & \multicolumn{2}{|c|}{$\begin{array}{l}\text { Iron sucrose, iv (Venofer; } \\
\text { Luitpold Pharmaceuticals) } \\
100 \text { mg thrice weekly for up } \\
\text { to } 6 \text { doses or until ICU discharge }\end{array}$} & \multicolumn{2}{|c|}{$\begin{array}{l}\text { Iron saccharate, iv (Venofer; } \\
\text { Vifor) } 20 \mathrm{mg} \text { and iv folic acid } \\
1 \mathrm{mg} \text { daily from day } 1-14^{(\mathrm{b})} \\
\text { Co-intervention: folic acid } \\
1 \mathrm{mg} \text { daily }\end{array}$} \\
\hline
\end{tabular}

and postoperatively and during 
Table 1 Characteristics of included studies $(n=5)$ (Continued)

Reported outcomes (follow-up • Hb concentration (baseline, operating $• H b$ concentration (day 0, time points, days)

room entry (day 7), exit operating day $1-5$, day 15 , day 30$)$
1 month after discharge, and

intravenous placebo while

hospitalised

Comparator

Oral and iv placebo pre-operatively and postoperatively following same protocol. room, ICU admission, ICU discharge, postoperative day 10 and day 30 post-hospital hospital discharge) - Immature reticulocyte fraction, - Reticulocyte counts (day 1 , day 5 , day 15 , day 30 ) - Serum ferritin (day 0, ay

reticulocyte count, serum ferritin (day $\cdot$ RBC transfusion 1 , postoperative day 10 and day 30 - Mortality post-hospital discharge)

- RBC transfusion (number of patients transfused, location of transfusion, mean number of units)
Oral placebo, same schedule as intervention protocol.

Placebo, iv $(100 \mathrm{~mL}$ of $0.9 \%$ saline) similar dosing schedule

No iron Co-intervention folic Co-intervention: ascorbic acid $500 \mathrm{mg}$ thrice daily, cyanocobalamin $1 \mathrm{mg}$, folic acid $1 \mathrm{mg}$

- Difference in Hct (baseline, days 7, 14, 21 and 28) (primary outcome) - Serum iron, serum ferritin, eZPP (baseline, day 7, day 14 , day 21 , day 28) - RBC transfusion - Estimated blood loss per study day

- Nosocomial infection

- Antibiotic days

- Adverse outcomes gastrointestinal upset - Mo and hospital LOS

- Mortality
- Number of total doses of study drug received - Hb concentration (daily) serum iron, serum ferritin, day 7 , day 14) - RBC transfusions

- Transfusion-free days

- Nosocomial infection and type - Antibiotic exposure

- ICU and hospital LOS

- Mortality 14, 21) (days 0, 7, 14, 21)

- Serum EPO (days 0, 2, 6 10, 21)

- Serum iron, transferrin Tsat, ferritin, eZPP CRP

(days 0, 10, 21)

- Mean blood loss (day 0-21)

- RBC transfusion (mea number of units) (day 0-21)

- ICU LOS

- Mortality
- Hb concentration (days 0, 7,

- Reticulocyte count, sTfR

an a second intervention arm, patients received intravenous iron and recombinant-human erythropoietin (EPO) (300 IU/kg) subcutaneously (s/c) on day 1; this treatment arm was not included in this review because the co-intervention was not matched in the control group. 'In a second intervention arm, patients received intravenous iron and EPO alfa (300 IU/kg) s/c on days 1, 3, 5, 7, 9; this treatment arm was not included in this review because the co-intervention was not matched in the control group. ICU intensive care unit, Hb haemoglobin, Hct haematocrit, Tsat transferrin saturation, sTfR soluble transferrin receptor, eZPP erythrocyte zinc protoporphyrin, $L O S$ length of stay, CBP cardiopulmonary bypass, $R B C$ red blood cell, $C r C l$ creatinine clearance, IE infective endocarditis 


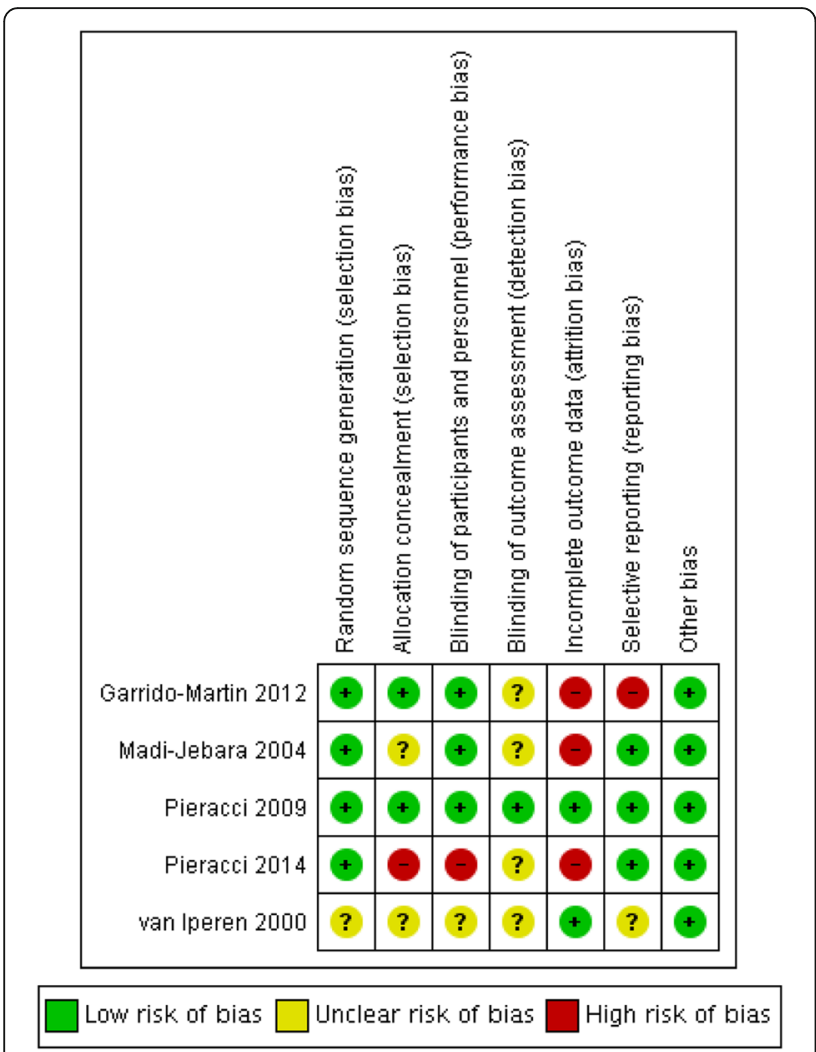

Fig. 2 Risk of bias summary showing review authors' judgements about each risk of bias item for each included study

trials that did not report mean and SD values, in one trial of oral iron supplementation there was no difference in serum ferritin between iron and placebo groups throughout the study period [22] and in one trial of intravenous iron supplementation there were significantly higher serum ferritin levels in the iron group compared to the placebo group at day 7 (median $808 \mathrm{ng} / \mathrm{mL}$ vs. $457 \mathrm{ng} / \mathrm{mL}, p<0.01$ ) and day 14 (median $1,046 \mathrm{ng} / \mathrm{mL}$ vs. $551.5 \mathrm{ng} / \mathrm{mL}, p<0.01$ ) [23].

Three trials reported serum iron and eZPP measurements [22-24], all of which found no significant difference in concentrations between iron and placebo groups at any time point. Meta-analysis was precluded by a lack of reporting of mean and SD values. Two trials reported Tsat levels - in one trial there was a statistically significant increase in Tsat levels at day 7 in the intravenous iron group when compared to placebo $(15 \%$ vs. $11 \%$, respectively, $p=0.02$ ) but this was below the normal range and did not persist through to the end of the study period [21], whereas in another trial there were no differences in Tsat levels throughout the study period [24].

\section{Other secondary outcomes}

No trials reported data on morbidity scales, HrQoL scores or serious adverse events as per the definition above.

\section{Adverse events}

Only one trial [22] comparing oral iron to placebo reported adverse effects - namely gastrointestinal upset defined as "nausea, vomiting, abdominal cramping, or constipation, necessitating discontinuation of the study drug for any period of time". The authors found no difference between the iron and placebo groups $(12.4 \%$ vs. $8.7 \%$ respectively, $p=0.62$ ).

\section{Discussion}

This systematic review identified five randomised trials enrolling a total of 665 randomised participants to evaluate the effect of iron as a treatment for anaemia in critical care. There was no evidence that iron supplementation to treat anaemia in adult critical care reduced allogeneic $\mathrm{RBC}$ transfusion requirements or increased mean haemoglobin concentrations, and there was no evidence of an impact on mortality and length of stay. Serum ferritin concentrations appeared to be significantly elevated in patients who receive iron supplementation at both shortterm and mid-term follow up. No studies reported on HrQoL. However, the available evidence from randomised trials of iron supplementation in critical care patients is incomplete, and the confidence intervals for all outcomes were wide, and these limits could encompass clinically important differences. Only one trial was graded at low-risk across all domains.

Across all outcomes the analyses in our review showed moderate heterogeneity between studies due to differences in patient population, the intervention and the intervention dose. The patient populations varied across all trials - cardiac, general surgical, trauma, neurosurgical and medical - limiting the external generalizability of the data. The majority of the patients included were in surgical ICUs. There was widespread variation in the dosing regimens of iron and methods of administration. The effect of oral iron may be reduced due to poor absorption in patients with impaired gastro-intestinal function - particularly in surgical/trauma patients. Intravenous iron may be more effective than oral iron in states of inflammation, as it can bypass the effects of hepcidin, an inhibitor of enteric absorption [11], but the four trials evaluating intravenous iron tested different formulations and schedules. Finally, the follow-up time points in all included studies were relatively short - the longest duration was up to 42 days, so any potential long-term clinical benefits of iron therapy may have been missed.

To our knowledge, this is the first systematic review of iron therapy in adult critical care. Strengths include a comprehensive literature search repeated at two time points, duplicate data extraction, and consideration of a broad range of biochemical and clinical outcomes. A multicentre, phase IIb, randomised, placebo-controlled trial in critically ill patients, comparing intravenous iron 


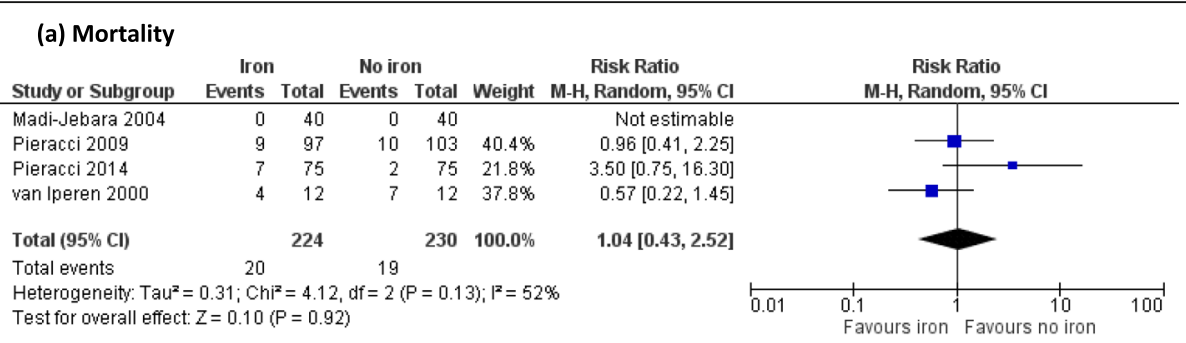

(b) In-hospital infection

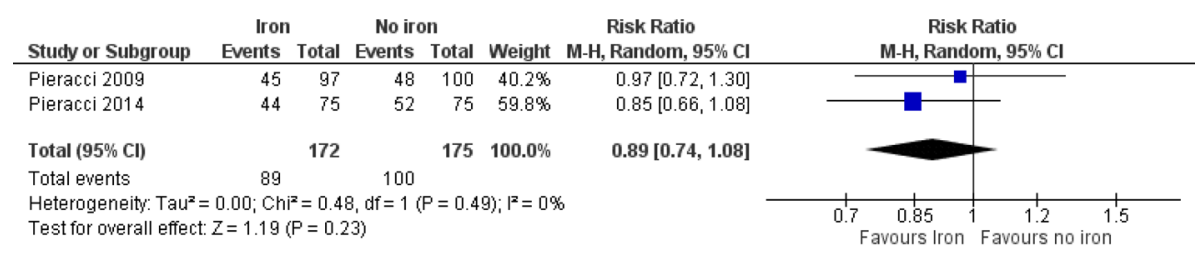

(c) Serum Ferritin $(\mathrm{ng} / \mathrm{mL})(\leq 10$ days)

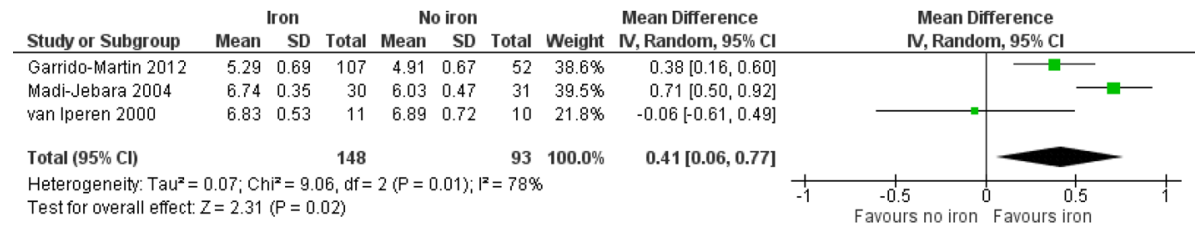

(d) Serum Ferritin (ng/mL) (>10 days)

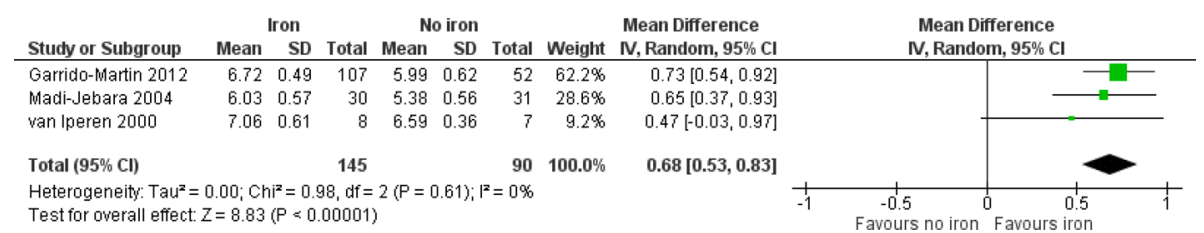

Fig. 5 Effect of iron supplementation, by any route, on secondary outcomes a mortality $\mathbf{b}$ in-hospital infection and serum ferritin at c short-term follow-up and $\mathbf{d}$ medium-term follow-up. $\mathrm{Cl}$ confidence interval, $\mathrm{M}$-H Mantel-Haenszel test, $\mathrm{N}$ inverse variance

in addition to standard care with standard care alone is currently recruiting (ACTRN 12612001249842) [25], and will be incorporated into updated versions of this review.

Our systematic review should be interpreted alongside other recent reviews. However, these reviews provide little data specific to iron supplementation in critically ill patients. For example, a recent meta-analysis on the safety and efficacy of intravenous iron in reducing requirement for allogeneic blood transfusion across all patient groups suggests iron is effective in reducing the requirement for allogeneic RBC transfusion and increasing haemoglobin concentration, but this meta-analysis did not report subgroup analyses in critical care patients. The apparent benefit of iron reported in this review was also counterbalanced by an increased risk of infection [11]. There was no difference, however, in serious adverse events or mortality in those receiving intravenous iron compared with oral or no iron. Similarly, a systematic review [26] investigating the safety and efficacy of iron therapy (oral and/or intravenous) for the treatment of anaemia in adults (including critical care patients but without subgroup analysis) found that both oral and intravenous iron reduced the need for blood transfusion. Intravenous iron was more effective at improving haemoglobin levels and quality of life, but neither route reduced mortality.

Any potential advantages of iron therapy must be balanced against the risk of adverse events. The risk of infection with use of iron is a significant concern in critically ill patients. Free iron has been shown to promote bacterial growth in vitro [27] and a meta-analysis [11] reported that intravenous iron was associated with a significant risk of infection. In our review only one trial reported on adverse events, and two trials reported on infection outcomes, with no significant differences. The two trials included in this study that reported infection outcomes used the same standardized definitions for nosocomial infection. However, infection is often not an agreed predefined endpoint in many studies [11], which can limit the strength of the conclusions.

The characterisation of anaemia in critical care patients is currently challenging as the tests routinely used 
to screen for iron deficiency, such as ferritin and transferrin, are confounded by the presence of inflammation. The recent discovery of the hormone hepcidin, a key regulator of iron metabolism and inhibitor of enteric iron absorption, has opened up avenues for improved diagnosis and management of anaemia in patients with concomitant inflammation such as those with critical illness [28]. It has been postulated that hepcidin may provide a better marker of iron deficiency than the routine biochemical tests in current use, thereby allowing more precise identification of patients with anaemia who likely to respond to iron therapy, whether intravenous or oral. The concept of using serum hepcidin to identify iron deficiency in the context of inflammation has been successfully applied to other patient populations where it has also predicted responsiveness to iron therapy $[29,30]$. Whether this holds true for critically ill patients requires further investigation. Furthermore, an understanding of the biological role of hepcidin in iron mobilisation might suggest that intravenous iron may be more advantageous as part of a targeted strategy to manage the iron-deficiency component of anaemia in critically ill patients, in whom serum hepcidin would be elevated and oral iron absorption therefore suppressed.

\section{Conclusion}

This review has identified five small trials in a diverse group of critically ill patients. On the basis of lowmoderate quality evidence, we have shown that there is no evidence that iron supplementation, either intravenous or enteral, to treat anaemia in critically ill patients reduces transfusion requirements or the mean number of RBCs transfused, or increases haemoglobin concentration. However the strength of this conclusion is limited by heterogeneity between studies and risks of bias. It is clear that further well-designed prospective studies in larger numbers of patients, with clinically relevant outcomes and taking into account many of the limitations identified by this review, are required to understand the role of iron supplementation in critical care. At present, the findings do not support any specific strategy for routine administration of iron, by any route, to treat in patients with anaemia in adult critical care.

\section{Additional file}

Additional file 1: Search strategy. Search narrative first performed in March 2015 and repeated on 9 March 2016. (DOCX 37 kb)

\section{Abbreviations}

Cl: confidence interval; EPO: erythropoietin; eZPP: erythrocyte zinc protoporphyrin; HDU: high-dependency unit; HrQoL: health-related quality of life; ICU: intensive care unit; IV: intravenous; LOS: length of stay; MD: mean difference; MODS: multiple organ dysfunction score; PRISMA: preferred reporting items for systematic reviews and meta-analyses; RBC: red blood cell;
RCT: randomized controlled trial; SD: standard deviation; SOFA: sequential organ failure assessment; Tsat: transferrin saturation

\section{Acknowledgements}

This research was supported by National Health Service (NHS) Blood and Transplant. The views expressed in this publication are those of the authors and not necessarily those of the NHS or the Department of Health.

\section{Funding}

No external funding was required for this research.

\section{Availability of data and materials}

All data generated or analysed during this study are included in this published article and its supplementary information files.

\section{Authors' contributions}

AS, SAF, SJS and NR contributed substantially to study design, data analysis and interpretation. CD performed the search strategies. AS and NR performed data extraction. AS and SAF performed data analysis and interpretation. AS drafted the manuscript, and NR, SM, CD, SAF and SJS revised it critically. All authors have read and approved the final version of the manuscript.

\section{Competing interests}

The authors declare that they have no competing interests.

\section{Consent for publication}

Not applicable.

Ethics approval and consent to participate

Not applicable.

\section{Author details}

${ }^{1}$ Nuffield Department of Anaesthetics, Level 2 John Radcliffe Hospital, Oxford University Hospitals NHS Foundation Trust, Oxford, UK. ${ }^{2}$ Weatherall Institute of Molecular Medicine, University of Oxford, Oxford, UK. ${ }^{3}$ Systematic Review Initiative, NHS Blood \& Transplant, Oxford, UK. ${ }^{4} \mathrm{NHS}$ Blood and Transplant, Oxford University Hospitals NHS Foundation Trust, John Radcliffe Hospital, Oxford, UK. ${ }^{5}$ Radcliffe Department of Medicine, University of Oxford, Oxford, UK.

Received: 21 June 2016 Accepted: 13 September 2016

Published online: 29 September 2016

\section{References}

1. Walsh TS, Wyncoll DA, Stanworth SJ. Managing anaemia in critically ill adults. BMJ. 2010;341:547-51.

2. Vincent JL, Baron JF, Reinhart K, Gattinoni L, Thijs L, Webb A, MeierHellmann A, Nollet G, Peres-Bota D. ABC (Anemia and Blood Transfusion in Critical (are) Investigators: anemia and blood transfusion in critically ill patients. JAMA. 2002;288:1499-507.

3. Walsh TS, Lee RJ, Maciver CR, et al. Anemia during and at discharge from intensive care: the impact of restrictive blood transfusion practice. Intensive Care Med. 2006;32:100-9.

4. Westbrook A, Pettila V, Nichol A, Blood Observational Study Investigators of ANZICS Clinical Trials Group, et al. Transfusion practice and guidelines in Australian and New Zealand intensive care units. Intensive Care Med. 2010; 36:1138-46

5. Walsh TS, Garrioch M, Maciver C, Audit of Transfusion in Intensive Care in Scotland Study, et al. Red cell requirements for intensive care units adhering to evidence-based transfusion guidelines. Transfusion. 2004;44: 1405-11.

6. McQuilten ZK, Schembri N, Polizotto MN, et al. Hospital blood bank information systems accurately reflect patient transfusion: results of a validation study. Transfusion. 2011;51:948-68.

7. Murphy GJ, Reeves BC, Rogers CA, Rizvi SI, Culliford L, Angelini GD. Increased mortality, postoperative morbidity, and cost after red blood cell transfusion in patients having cardiac surgery. Circulation. 2007:116:2544-52.

8. Isbister JP, Shander A, Spahn DR, Erhard J, Farmer SL, Hofmann A. Adverse blood transfusion outcomes: establishing causation. Transfus Med Revs. 2011;25:89-101. 
9. Marik PE, Corwin HL. Efficacy of red blood cell transfusion in the critically ill: a systematic review of the literature. Crit Care Med. 2008:36:2667-74.

10. Hayden SJ, Albert TJ, Watkins TR, Swenson ER. Anemia in critical illness: insights into etiology, consequences and management. Am J Resp Crit Care Med. 2012;185:1049-57.

11. Litton E, Xiao J, Ho KM. Safety and efficacy on intravenous iron therapy in reducing requirement for allogeneic blood transfusion: a systematic review and meta-analysis of randomised clinical trials. BMJ. 2013;347:f4822.

12. Shah A, Roy N, McKechnie S, Stanworth SJ, Doree C, Fisher S. Iron supplementation to treat anaemia in adult critical care patients. PROSPERO 2015: CRD42015016627. Available from http://www.crd.york.ac.uk/ PROSPERO/display_record.asp?ID=CRD4201501662. Accessed 2 Mar 2015.

13. Albaramki J, Hodson EM, Craig JC. Parenteral versus oral iron therapy for adults and children with chronic kidney disease. Cochrane Database Syst Rev. 2012:1:CD007857.

14. Reveiz L, Gyte GML, Cuervo LG, Casasbuenas A. Treatments for iron-deficiency anaemia in pregnancy. Cochrane Database Syst Rev. 2011;10:CD003094.

15. International Conference on Harmonisation (ICH) of Technical Requirements for Registration of Pharmaceuticals for Human Use. Code of Federal Regulation \& ICH Guidelines. Media: Parexel Barnett; 1996.

16. Higgins JPT, Altman DG. Assessing risk of bias in included studies. In: Higgins JPT, Green S, editors. Cochrane Handbook for Systematic Reviews of Interventions. Chichester: John Wiley \& Sons; 2008. p. 187-241.

17. Review Manager (RevMan) [Computer program]. Version 5.3. Copenhagen: The Nordic Cochrane Centre, The Cochrane Collaboration; 2014

18. Higgins JPT, White IR, Anzures-Cabrera J. Meta-analysis of skewed data: Combining results reported on log-transformed or raw scales. Stat Med. 2008;27:6072-92

19. Garrido-Martin P, Nassar-Mansur Ml, Llana-Ducros R, et al. The effect of intravenous and oral iron administration of perioperative anaemia and transfusion requirements in patients undergoing elective cardiac surgery: a randomized clinical trial. Interact Cardiovasc Thorac Surg. 2012;15:1013-8.

20. Higgins JT, Thompson SG, Deeks JJ, Altman DG. Measuring inconsistency in meta-analyses. BMJ. 2003;327:557-60.

21. Madi-Jebara S, Sleilaty GS, Achouch PE, et al. Postoperative intravenous iron used alone or in combination with low-dose erythropoietin is not effective for correction of anemia after cardiac surgery. J Cardiothorac Vasc Anesth. 2004;18:59-63.

22. Pieracci FM, Henderson P, Rodney JR, et al. Randomized, double-blind, placebo-controlled trial of effects of enteral iron supplementation on anemia and risk of infection during surgical critical illness. Surg Infect (Larchmt). 2009:10:9-19.

23. Pieracci FM, Stovall RT, Jaouen B, et al. A Multicentre, Randomized Clinical Trial of IV Iron Supplementation for Anemia of Traumatic Critical IIIness. Crit Care Med. 2014;42:2048-57.

24. van Iperen CE, Gaillard CAJM, Kraaijenhagen RJ, et al. Response of erythropoiesis and iron metabolism to recombinant human erythropoietin in intensive care unit patients. Crit Care Med. 2000;28:2773-8.

25. Litton E, Baker S, Erber W, et al. The IRONMAN trial: a protocol for a multicentre randomised placebo-controlled trial of intravenous iron in intensive care unit patients with anaemia. Crit Care Resusc. 2014;16:285-90.

26. Clevenger C, Gurusamy K, Klein AK, et al. Systematic review and meta-analysis of iron therapy in anaemic adults without chronic kidney disease: updated and abridged Cochrane review. Eur J Heart Fail. 2016. doi:10.1002/ejhf.514.

27. Fishbane S. Review of issues relating to iron and infection. Am J Kidney Dis. 1999;34 Suppl 2:547-52.

28. Goodnough LT. Iron deficiency syndromes and iron restricted erythropoiesis. Transfusion. 2012;52:1584-92.

29. Pasricha SR, Atkinson SA, Armitage AE, et al. Expression of the Iron Hormone Hepcidin Distinguishes Different Types of Anemia in African Children. Sci Transl Med. 2014:6:235re3.

30. Bregman DB, Morris D, Koch TA, He A, Goodnough LT. Hepcidin levels predict nonresponsiveness to oral iron therapy in patients with iron deficiency anaemia. Am J Hematol. 2013;88:97-101.

\section{Submit your next manuscript to BioMed Central and we will help you at every step:}

- We accept pre-submission inquiries

- Our selector tool helps you to find the most relevant journal

- We provide round the clock customer support

- Convenient online submission

- Thorough peer review

- Inclusion in PubMed and all major indexing services

- Maximum visibility for your research

Submit your manuscript at www.biomedcentral.com/submit
Biomed Central 